\title{
Black hole evaporation in a thermalized final-state projection model
}

\author{
A. Fabbri and A. Pérez \\ Departamento de Física Teórica and IFIC, Universidad de Valencia-CSIC, Dr. Moliner 50, 46100 Burjassot, Spain
}

(Received 21 November 2006; published 12 March 2007)

\begin{abstract}
We propose a modified version of the Horowitz-Maldacena final-state boundary condition based upon a matter-radiation thermalization hypothesis on the Black Hole interior, which translates into a particular entangled state with thermal Schmidt coefficients. We investigate the consequences of this proposal for matter entering the horizon, as described by a Canonical density matrix characterized by the matter temperature $T$. The emitted radiation is explicitly calculated and is shown to follow a thermal spectrum with an effective temperature $T_{\text {eff }}$. We analyze the evaporation process in the quasistatic approximation, highlighting important differences in the late stages with respect to the usual semiclassical evolution, and calculate the fidelity of the emitted Hawking radiation relative to the infalling matter.
\end{abstract}

DOI: 10.1103/PhysRevD.75.064009

PACS numbers: 04.70.Dy, 03.67.-a

Black holes (BHs) are probably the most fascinating objects in our Universe. Although initially related to the general theory of relativity, they have become an interdisciplinary field, where ideas from thermodynamics, quantum field theory in curved spacetimes, string theory and quantum information are applied in order to understand those aspects which go beyond the purely gravitational context. Among them, the most spectacular is the BH evaporation effect [1], which shows that quantum $\mathrm{BHs}$, unlike their classical counterparts, emit particles in the form of thermal radiation. Even more intriguing is the suggestion Hawking made [2] that black holes will evaporate completely and the information about their formation will be lost forever. Obviously, if the whole process is governed by a unitary transformation, as demanded by quantum mechanics, no information can be lost. This question, however, is still under debate, and several hypothesis have been suggested [3].

Recently, Horowitz and Maldacena [4] (HM, hereafter) have made a proposal to describe this transformation based on a final-state projection condition, which resembles quantum teleportation (but without a classical communication channel). In the original HM proposal, matter inside the $\mathrm{BH}$ is in a maximally entangled state with the incoming Hawking radiation. The unknown effects of quantum gravity are encoded into an additional unitary transformation $S$ acting on matter states alone. The overall transformation acting on matter entering the horizon is obtained by the projection onto this final state.

Several comments and modifications of this interesting proposal have been discussed later. Gottesmann and Preskill [5] have argued that the interaction between the collapsing body and the infalling Hawking radiation would modify the suggested maximally entangled state, and this modification gives rise, in general, to a loss of information. Following this idea, in [6] the author allows for a modified final state with random Schmidt coefficients. As a consequence, some information is lost, giving "almost certain escape" for general states.
In this paper, we propose a different form for the HM entangled state, which is in fact described by a thermal spectrum. Several arguments will be given to justify this proposal. We will then determine explicitly the radiation emitted by the $\mathrm{BH}$, and analyze both the consequences regarding the evaporation process and the relation between the information contained in the infalling matter and that of the emitted Hawking radiation. We use units such that $G=$ $\hbar=c=k_{B}=1$.

Let us begin with a brief description of the final-state projection proposal. The Hilbert space of the infalling matter plus Hawking radiation can be written as

$$
H=H_{M} \otimes H_{\text {in }} \otimes H_{\text {out }},
$$

where $H_{M}$ corresponds to matter degrees of freedom, and $H_{\text {in }}\left(H_{\text {out }}\right)$ stands for the incoming (outgoing) Hawking radiation, described by an entangled thermal state belonging to $H_{\text {in }} \otimes H_{\text {out }}$. We denote this state by $|\phi\rangle_{\text {in } \otimes \text { out }}$ and can be expressed, in a compact form, as

$$
|\phi\rangle_{\text {in } \otimes \text { out }}=\sum_{j} \lambda_{j}^{T_{H}}|j\rangle_{\text {in }} \otimes|j\rangle_{\text {out }} .
$$

In the above equation, the states $|j\rangle_{\text {in }}\left(|j\rangle_{\text {out }}\right)$ denote Fock states for the incoming (outgoing) radiation. In a more detailed way, we can write

$$
|j\rangle_{\text {in }}=\left|N_{1} N_{2} \ldots\right\rangle_{\text {in }}
$$

(analogously for $|j\rangle_{\text {out }}$ ), where $N_{i}$ denotes the occupation number for frequency $\omega_{i},(i=1,2, \ldots)$ and we have to take into account all possible positive frequencies. Following these conventions, the coefficients in Eq. (2) would read as

$$
\lambda_{j}^{T_{H}}=\frac{1}{\sqrt{Z_{H}}} \exp \left(-\beta_{H} \epsilon_{j} / 2\right),
$$

with $\epsilon_{j}$ the energy corresponding to the Fock state (3), $\beta_{H}=1 / T_{H}$ the inverse of the Hawking temperature, given, for the Schwarzschild $\mathrm{BH}$, by $T_{H}=1 / 8 \pi M$ ( $M$ is its mass), and $\frac{1}{\sqrt{Z_{H}}}$ is a normalization factor. 
Following HM, the matter state inside the $\mathrm{BH}$, together with the infalling radiation, form a pure entangled state $|\psi\rangle_{M \otimes \text { in }}$, written in the form

$$
|\psi\rangle_{M \otimes \text { in }}=\sum_{j} \lambda_{j}|j\rangle_{M} \otimes|j\rangle_{\text {in }}
$$

The states $\left\{|j\rangle_{M}\right\}$ form an orthonormal basis of the Hilbert space $H_{M}$. In the original proposal, it is suggested that this state is maximally entangled, therefore all the $\lambda_{j}$ would be the same. Moreover, as mentioned above, a BH unitary transformation $S$ acting on matter degrees of freedom is included in that proposal. We will, however, keep the above form, with yet undefined coefficients $\lambda_{j}$, as a starting point for our discussion.

Let us first compare Eqs. (2) and (5). From each of them one can obtain the density matrix describing the infalling Hawking radiation, by tracing out over the remaining degrees of freedom. For consistency, both expressions should coincide, i.e.:

$$
\rho_{\text {in }}=\operatorname{tr}_{\text {out }}\left(|\phi\rangle_{\text {in } \otimes \text { out in } \otimes \text { out }}\langle\phi|\right)=\operatorname{tr}_{M}\left(|\psi\rangle_{M \otimes \text { in } M \otimes \text { in }}\langle\psi|\right) .
$$

In other words,

$$
\rho_{\text {in }}=\sum_{j}\left(\lambda_{j}^{T_{H}}\right)^{2}|j\rangle_{\text {inin }}\left\langle j\left|=\sum_{j}\left(\lambda_{j}\right)^{2}\right| j\right\rangle_{\text {inin }}\langle j|,
$$

which implies $\lambda_{j}=\lambda_{j}^{T_{H}} \forall j$. As a consequence, the state $|\psi\rangle_{M \otimes \text { in }}$ described by Eq. (5) adopts the form of a thermal state, similar to Eq. (2). More precisely, if we trace out the incoming radiation, the resulting density matrix is given by

$$
\rho_{M}=\operatorname{tr}_{\text {in }}\left(|\psi\rangle_{M \otimes \text { in } M \otimes \text { in }}\langle\psi|\right)=\sum_{j}\left(\lambda_{j}^{T_{H}}\right)^{2}|j\rangle_{M M}\langle j|,
$$

where the diagonal elements $\left(\lambda_{j}^{T_{H}}\right)^{2}$ follow a (Canonical) thermal distribution with temperature equal to the Hawking temperature. Let us discuss this result in more detail. One could argue that, as matter enters the horizon, it would eventually thermalize with the incoming radiation, and finally adopt a thermal distribution with the same temperature (the Hawking temperature). A supporting argument for this hypothesis is given in [7], where it is claimed that, as a spacelike singularity is approached, solutions to Einstein's equations become chaotic, with rapid cycles through all states in the Hilbert space, so that time averages give the same results as ensemble averages on a thermal system.

We also note that the above suggested (thermal) form for $|\psi\rangle_{M \otimes \text { in }}$ has also been discussed in [8] within the context of gravitational collapse of a matter shell (described by a scalar massless field). It is then shown that, for a given frequency $\omega$, the stationary state of matter and incoming radiation inside the black hole is a "maximally entangled two-mode squeezed" state with coefficients given by Eq. (4). However, when discussing the final-state projec- tion, in this reference the author also includes the unknown $S$ matrix discussed in the HM proposal. Our point of view is different: given the arguments above, we suggest the state $|\psi\rangle_{M \otimes \text { in }}$ as the state to be used for the final-state projection. In this way, our proposal resembles the one in [6] (compare Eq. (6) in this reference with our Eq. (5)), but now the $\lambda_{j}$ 's to be used, instead of possessing a random distribution, have a thermal one, shown in (4). Notice that the ideas we introduce in this paper specifies the coefficients appearing in (5), without modifying the nonlinear nature of the HM proposal. The consequences of such a nonlinear behavior, when a subset of a bipartite system crosses the horizon, while the other part remains coherently accessible to experiments in the exterior region, have been studied in [9].

This specific form for the final state allows us to make explicit calculations for the emitted radiation within the context of final-state projection, as suggested by HM. Following these ideas, we define the projector

$$
\Lambda_{M \otimes \text { in }}=|\psi\rangle_{M \otimes \text { in } M \otimes \text { in }}\langle\psi| .
$$

An incoming state of matter $|\chi\rangle_{M}$ entering the horizon would be transformed into the state $|\phi\rangle_{\text {out }}$ of outgoing Hawking radiation, according to the projection

$$
\Lambda_{M \otimes \text { in }}\left(|\chi\rangle_{M}|\phi\rangle_{\text {in } \otimes \text { out }}\right)=|\psi\rangle_{M \otimes \text { in }}|\phi\rangle_{\text {out }} .
$$

In order to study more realistic situations, we generalize the above rule to matter described by a density matrix. This would be the case, for example, when we consider a nondegenerate gas falling into the black hole, as we discuss later. Let $\rho_{M}$ be the matter density matrix, and define $\rho_{\text {in } \otimes \text { out }}=|\phi\rangle_{\text {in } \otimes \text { outin } \otimes \text { out }}\langle\phi|$. We extend the final-state projection in the straightforward way

$$
\Lambda_{M \otimes \text { in }}\left(\rho_{M} \otimes \rho_{\text {in } \text { out }}\right) \Lambda_{M \otimes \text { in }} \equiv|\psi\rangle_{M \otimes \text { in } M \otimes \text { in }}\langle\psi| \otimes \bar{\rho}_{\text {out }},
$$

where $\bar{\rho}_{\text {out }} \equiv_{M \otimes \text { in }}\left\langle\psi\left|\left(\rho_{M} \otimes \rho_{\text {in } \otimes \text { out }}\right)\right| \psi\right\rangle_{M \otimes \text { in }}$ is the unnormalized density matrix describing the outgoing Hawking radiation. After some algebra, one obtains the following result for the normalized density matrix:

$$
\rho_{\text {out }}=\frac{\sum_{i, j} \rho_{M i j} \lambda_{i}^{T_{H}} \lambda_{j}^{T_{H}}|i\rangle_{\text {outout }}\langle j|}{\sum_{i} \rho_{M i i}\left(\lambda_{i}^{T_{H}}\right)^{2}},
$$

with $\rho_{M i j}={ }_{M}\left\langle i\left|\rho_{M}\right| j\right\rangle_{M}$ the matrix elements of $\rho_{M}$. As a particular case, which will be useful for further discussions, let us assume that the infalling matter is described by a Canonical ensemble characterized by a temperature $T$, such that $\rho_{M}=\frac{1}{Z} \sum_{i} e^{-\beta \epsilon_{i}}|i\rangle_{M M}\langle i|$ and $\beta=1 / T$. Here $Z=\sum_{i} e^{-\beta \epsilon_{i}}$ is the matter partition function. In this case, it is straightforward to obtain

$$
\rho_{\text {out }}=\frac{1}{Z_{\text {eff }}} \sum_{i} e_{i}^{-\beta_{\text {eff }} \epsilon_{i}}|i\rangle_{\text {outout }}\langle i|,
$$


where $\beta_{\text {eff }}=\beta+\beta_{H}$ and $Z_{\text {eff }} \equiv \sum_{i} e_{i}^{-\beta_{\text {eff }} \epsilon_{i}}$. From the above equation, we immediately see that, as a consequence of the model introduced in this paper, the $\mathrm{BH}$ radiates with an effective temperature

$$
T_{\text {eff }}=\frac{T T_{H}}{T+T_{H}} .
$$

Suppose we consider the infall of matter inside a $\mathrm{BH}$. This situation could correspond to the accretion disk around a solar mass (or larger) $\mathrm{BH}$. The gas can be described by a nondegenerate (Canonical) distribution function. The temperature of the gas in the inner disk depends on several parameters such as the mass of the $\mathrm{BH}$, the distance to the center and the viscosity of the gas, but typically one finds temperatures $T \sim 10^{7} K$ [10]. For $M \sim M_{\odot}$, the Hawing temperature is $T_{H} \sim 10^{-7} \mathrm{~K}$. Since $T \gg T_{H}$ the black hole emits radiation at the usual Hawking temperature $T_{\text {eff }} \sim T_{H}$. Using standard arguments, let us now consider the evaporation process modeled as a sequence of quasistationary states, each of them radiating with the instantaneous temperature $T_{\text {eff }}=$ $T_{\text {eff }}\left(T, T_{H}(M(t))\right)$, which varies with time, and where the BH mass $M(t)$ satisfies the evolution equation

$$
\frac{d M}{d t}=-4 \pi R_{S}^{2} \sigma T_{\text {eff }}^{4},
$$

with $R_{s}=2 M$ the Schwarzschild radius and $\sigma$ is the Stefan-Boltzmann constant. In the usual case $T_{\text {eff }} \rightarrow T_{H}$ and we have that the $\mathrm{BH}$ cooling time (corresponding to complete evaporation) is $t_{c}=\frac{256 \pi^{3}}{3 \sigma} M_{0}^{3}$, where $M_{0}=M(0)$ is the BH initial mass. Now, defining $m=M(t) / M_{0}$ and $\tau=t / t_{c}$ the above equation can be rewritten as

$$
\frac{d m}{d \tau}=-\frac{\gamma^{4} m^{2}}{3(1+\gamma m)^{4}},
$$

where $\gamma=T / T_{H}^{0}$ and $T_{H}^{0}=1 / 8 \pi M_{0}$ is the initial Hawking temperature.

Equation (15) shows two different regimes. The first one corresponds to $\gamma m=T / T_{H}(t) \gg 1$. During this phase one has $T_{\text {eff }} \simeq T_{H}$ and the evaporation proceeds as in the standard case, i.e. $m^{3} \simeq 1-\tau$. However, when $m \gamma \ll 1$, that is $T \ll T_{H}(t)$ and $T_{\text {eff }} \simeq T$, the evolution is drastically modified to $m \simeq 3 / \gamma^{4} \tau$, i.e. the mass goes to zero asymptotically, rather than showing a finite evaporation time.

For the example described above, one has $\gamma \sim 10^{14}$, which means that the transition $\gamma m \sim 1$ occurs at $\tau=$ $\tau_{1} \sim 1-10^{-42}$, when the BH mass is $M \sim M_{0} / \gamma \sim$ $10^{19} \mathrm{~g}$. Such a mass is certainly macroscopic, and well above the Planck mass scale, where quantum gravity effects are thought to dominate the evaporation. Equivalently, the transition corresponds to $T_{H} \sim T$. As we show below, at this stage the information released by the evaporating $\mathrm{BH}$ starts to approach the one corresponding to the initial infalling matter.
In order to compare how much information have in common the incoming matter and the outgoing radiation, we compute the fidelity $F\left(\rho_{\text {out }}, \rho_{M}\right)=\operatorname{tr}\left[\rho_{M}^{1 / 2} \rho_{\text {out }} \rho_{M}^{1 / 2}\right]^{1 / 2}$ between $\rho_{M}$ and $\rho_{\text {out }}$. Notice, however, that these two operators are expressed in different basis. Therefore, we first add the operator $U^{\prime}$ which performs the trivial, information conserving map $U^{\prime}|i\rangle_{M}=|i\rangle_{\text {out }}$. After some simple algebra, one obtains

$$
F\left(\rho_{\text {out }}, \rho_{M}\right)=\frac{1}{\left(Z Z_{\text {eff }}\right)^{1 / 2}} \sum_{j} e^{-\left(\beta+\beta_{\text {eff }}\right) \epsilon_{j} / 2} .
$$

It is simpler to take logs on the above expression. For example, one has $\log Z=-\sum_{\omega} \log \left(1-e^{-\beta \omega}\right)$. We perform the sum over frequencies by using a simple box normalization, i.e. $\sum_{\omega} \rightarrow V \int \frac{d^{3} \omega}{(2 \pi)^{3}}$, where $V$ is the box volume, which we take as $V=\frac{4}{3} \pi R_{s}^{3}$. The remaining terms in Eq. (16) are calculated in a similar way, giving the final expression

$$
\log F\left(\rho_{\text {out }}, \rho_{M}\right)=-\frac{24 y^{4}+48 y^{3}+33 y^{2}+9 y+1}{8640 y^{3}\left(2 y^{2}+3 y+1\right)^{3}} .
$$

The latter formula depends only on the ratio $y=T_{H} / T$.

The resulting expression, Eq. (17), is plotted in Fig. 1, as a function of $y$ for the most relevant range. As it follows from the above formula, $F\left(\rho_{\text {out }}, \rho_{M}\right)$ is exponentially suppressed for $T \gg T_{H}$, and becomes close to unity as $T_{H}$ approaches $T$. Let us return to the astrophysical scenario described previously. Initially, we have $y=1 / \gamma \sim 10^{-14}$; therefore, for similar scenarios $F\left(\rho_{\text {out }}, \rho_{M}\right) \rightarrow 0$, and the Hawking radiation does not contain the information about the infalling matter. Now assume that the accretion eventually stops, and that the $\mathrm{BH}$ continues to evaporate, so that $M$ decreases and $T_{H}$ increases accordingly. Following the above results, the information carried out by the accreted mass will be approximately recovered when $T \sim T_{H}$ or, in

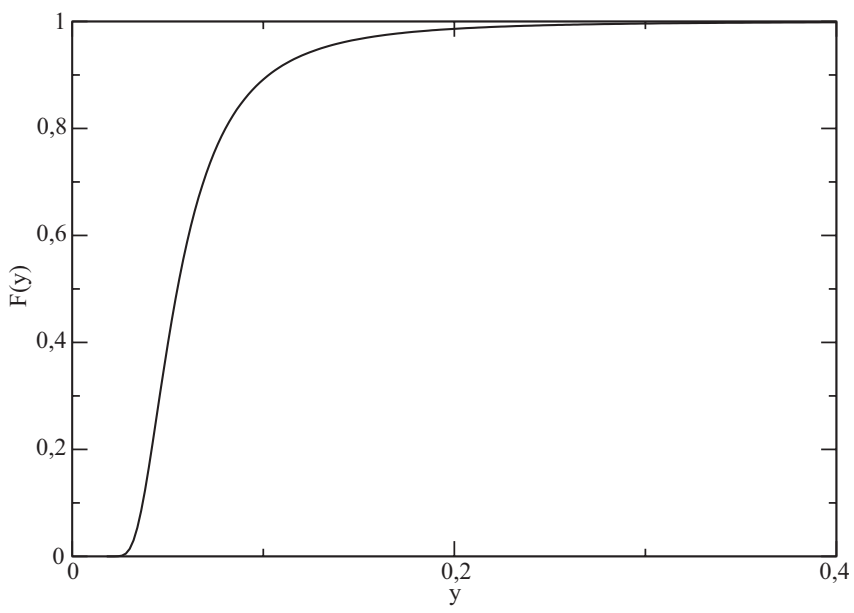

FIG. 1. Fidelity of $\rho_{\text {out }}$ with respect to $\rho_{M}$, as calculated from Eq. (17). 
other words, when the $\mathrm{BH}$ approaches the asymptotic evaporation phase.

To sum up the results presented in this paper, using a thermalized final-state projection model, slightly different from the original proposal by Horowitz and Maldacena, we considered the infall of matter (in the form of a thermal gas characterized by a temperature $T$ ) into the Black Hole. Our model allows to quantitatively determine the form of the radiation emitted by the Black Hole, which is thermal at the temperature $T_{\text {eff }}$ given in Eq. (13). Modeling the evaporation process as a sequence of quasistationary states, each characterized by the instantaneous temperature $T_{\text {eff }}$, we have shown that, for realistic values of the Black Hole initial mass $M_{0}$ and of $T$, the emission rate is the standard one, i.e. $T_{\text {eff }} \sim T_{H}$, for most of its lifetime.
Extrapolation of our results to the late stages of the evaporation shows the transition to a new regime, where $T_{\text {eff }}$ reaches its maximum value $T$ and the black hole mass goes to zero asymptotically. The fidelity, measuring the information content in the emitted radiation relative to the initial infalling matter, is essentially zero during the first phase of the evaporation, and goes to unity as the $\mathrm{BH}$ approaches the asymptotic regime, when information from the infalling matter would be released.

This work has been supported by the Spanish Grants AYA2004-08067-C01, FPA2005-00711, FIS2005-05736C03-03, the EU Network MRTN-CT-2004-005104 and by Generalitat Valenciana (Grant No. GV05/264).
[1] S. W. Hawking, Commun. Math. Phys. 43, 199 (1975).

[2] S. W. Hawking, Phys. Rev. D 14, 2460 (1976).

[3] See for instance: D. N. Page, Phys. Rev. Lett. 44, 301 (1980); S. B. Giddings, hep-th/9412138; T. Banks, A. Dabholkar, M. R. Douglas, and M. O'Loughlin, Phys. Rev. D 45, 3607 (1992); S. W. Hawking, Phys. Rev. D 37, 904 (1988); A. Strominger, hep-th/9501071; J. D. Bekenstein, gr-qc/9605059; 't Hooft, Nucl. Phys. B335, 138 (1990); L. Susskind, L. Thorlacius, and J. Uglum, Phys. Rev. D 48, 3743 (1993).

[4] G. T. Horowitz and J. Maldacena, J. High Energy Phys. 02
(2004) 008

[5] D. Gottesman and J. Preskill, J. High Energy Phys. 03 (2004) 026.

[6] S. Lloyd, Phys. Rev. Lett. 96, 061302 (2006).

[7] T. Banks and W. Fischler, hep-th/0606260.

[8] D. Ahn, Phys. Rev. D 74, 084010 (2006).

[9] U. Yurtsever and G. Hockney, Class. Quant. Grav. 22, 295 (2005).

[10] S. L. Shapiro and S. A. Teukolsky, Black Holes, White Dwarfs, and Neutron Stars (John Wiley and Sons, New York, 1983). 